\title{
MEASUREMENT OF RESIDUAL STRESSES WITH OPTICAL TECHNIQUES
}

\author{
João Ribeiro*, Jaime Monteiro***, Mário Vaz**, Hernâni Lopes*, Paulo Piloto* \\ * Instituto Politécnico de Bragança \\ Bragança, Portugal \\ Email: jribeiro@ipb.pt, hlopes@ipb.pt,ppiloto@ipb.pt \\ ** Faculdade de Engenharia da Universidade do Porto \\ Porto, Portugal \\ Email:gmavaz@fe.up.pt \\ *** Instituto de Engenharia Mecânica e Gestão Industrial \\ Leça do Balio, Portugal \\ Email:jmont@fe.up.pt
}

\begin{abstract}
The goal of this work is the development of other experimental techniques to measure residual stresses, as alternative to the hole-drilling method with strain gages. The proposed experimental techniques are based on the use of Moiré Interferometry and in-plane Electronic Speckle Pattern Interferometry (ESPI). Both are field techniques allowing the assessment of in-plane displacements without contact and high resolution. Grating replication techniques were developed to record high quality diffraction gratings onto the specimen's surfaces. An optical set-up of laser interferometry was developed to generate the master grating (virtual). An in-plane ESPI set-up was also designed and implemented to measure displacements in one direction. The stress relaxation was promoted by the blind hole-drilling and the obtained fringe patterns (Moiré and speckle) were video recorded. Image processing techniques were applied to assess the in-plane strain field. A finite elements code (ANSYS®) was used to simulate the stresses relaxation process whose values were compared with the experimental data and to calculate the hole-drilling calibration constants.
\end{abstract}

KEYWORDS: residual stresses, hole drilling, Moiré interferometry, ESPI, FEM.

\section{INTRODUCTION}

Residual stresses are the ones that remain on components and structures after the load has been removed. These may result from fabrication processes like welding and machining, or due to overload localized yielding. Usually these stresses are undesirables because they added to service stresses reducing the structures maximum load. However, when variable loads are expected compressions surface residual stresses are sometimes applied to improve fatigue resistance. This way the residual stress assessment assumes a very important role in mechanical design.

The most used technique for residual stress measurement is the hole drilling method with strain gages. In this case three discrete measurements were performed to obtain the stress released during the hole drill. Special rosettes and drilling devices are used for this propose according to ASTM standard [1]. This document describes the methodology and the limits of the measurements according to the expected in depth stress distribution. Other techniques are 
also available based on measurements of deformations at the crystallographic and macroscopic level.

Nowadays, image techniques have experienced a grate improvement in what concerns experimental mechanics applications. Displacement fields can be assessed by non-contact techniques with high resolution by using ordinary white light or laser illumination. Image correlation [2], geometric and interferometric Moiré [3], speckle or holographic interferometry [4], are available with resolutions up to the laser wave length.

In this work a new process to measure residual stresses with optical techniques (in-plane ESPI and Moiré Interferometry) was developed and combined with the hole-drilling method. Measurements were carried out on a ring and plug specimen, constructed to produce well known residual stress fields. The calibration coefficients were obtained by numerical simulation with a (FEM) code generated in the ANSYS® programme.

An image processing software package was developed by LOME (Laboratory of Optics and Experimental Mechanics) to calculate the displacement field from the interferometric patterns. Moiré Interferometry technique was used in the second test after replication of a grating with $1200 \mathrm{l} / \mathrm{mm}$ on the specimen surface. A set up to measure the in-plane displacement field in two directions was prepared and used.

\section{RESIDUAL STRESS MEASUREMENT SET-UPS}

In the current work two optical set-ups were prepared, one for Moiré Interferometry [5] and the other for double-illumination ESPI [6]. Both were used to measure the in-plane displacements generated by residual stress release. Image processing algorithms, involving filtering, phase calculation and unwrapping, and spatial differentiation were used in data postprocessing to transform surface displacement into residual stress fields. In fig. 1 a schematic presentation of the two optical set-ups used can be seen.

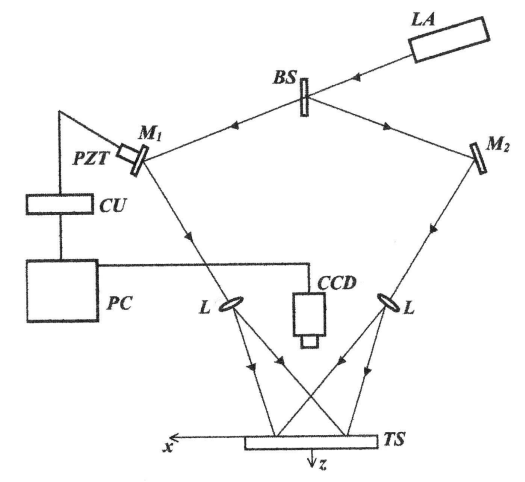

(a)

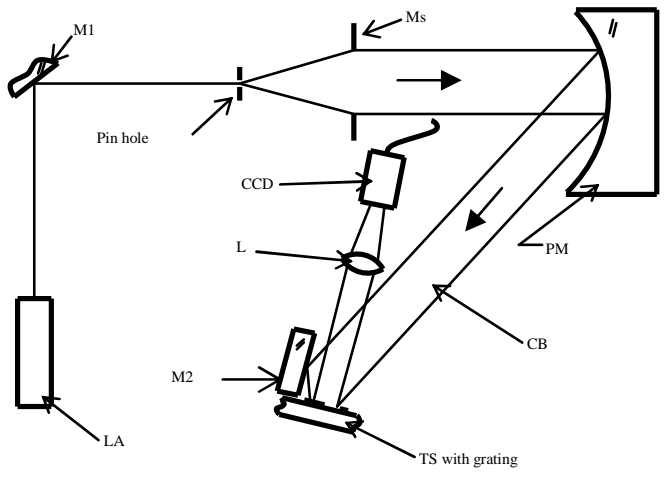

(b)

Fig.1 Schematic presentation of the optical set-ups used: (a) in plane ESPI [6]; (b) Moiré Interferometry [7].

Where LA is the laser source, BS is a beam splitter, M1 and M2 are plane mirrors, PZT is a piezoelectric phase modulation device, $\mathrm{L}$ is a lens, TS is the test specimen, Ms is a mask, CB is the collimated beam and PM is a parabolic mirror.

The stresses were released according to the conventional procedure used with strain gages. Drilling a hole on the surface of a specimen with residual stresses produces a stress relaxation around it. The corresponding deformation was assessed in this work with an optical 
technique. The stress analysis algorithms are based on the work of Nelson [8, 9] and Wu [10], adapted to work with ESPI and Moiré Interferometry data.

The light source used in both set-ups was a 2 watt laser from Coherent (Verdi). For image acquisition was selected a CCD Jai camera with four megapixel of resolution. A computer controlled PI piezoelectric actuator was used to perform the phase modulation needed to calculate the phase maps using the algorithm described by Creath [11]. Finally, a dedicated support was constructed with a $10 \mu \mathrm{m}$ resolution Microcontrol linear stage to allow depth control drilling. With this support the air turbine used as drill could be removed and replaced with minor chances in its alignment.

In the ESPI measurement an initial specklegram is acquired and saved. Then, the drill is placed in front of the test specimen and a small hole is drilled to a given depth. Next, the drill is removed and another specklegram is acquired. The interferogram resulting from the correlation of the two recordings leads to the surface displacements caused by the stress relaxation. In this case the surface information is codified in the speckle patterns.

For the Moiré Interferometry an high frequency grating is previously bonded on the surface of the specimen. The grating used was a 1200 lines/mm, obtained by aluminum vaporization on the top of an epoxy replication of a master grating from Photomechanis. The replication process followed is described by $\mathrm{Wu}$ [12]. The set-up proposed by Post [13] was used in the virtual grating generation by laser interferometry. The first recording was obtained by superposition of the virtual grating over the object replication grating. Then, a hole is drilled at the desired place, and the Moiré fringes due to stress relaxation are obtained and recorded. A tiltable parallel plate glass was used to promote phase modulation with a four image phase calculation algorithm [11].

In both cases, the residual stress field was computed establishing an appropriate stressdisplacement relationship by a Finite Element Method (FEM) code. According to Nelson [8] the displacements due to residual stresses relaxation are related between themselves through the following equations,

$$
u_{r}^{k}=\left[\begin{array}{r}
A+B \cos 2 \theta_{k} \\
A-B \cos 2 \theta_{k} \\
2 B \sin 2 \theta_{k}
\end{array}\right]^{-1}\left[\begin{array}{l}
\sigma_{x x} \\
\sigma_{y y} \\
\tau_{x y}
\end{array}\right]
$$

$$
u_{\theta}^{k}=\left[\begin{array}{r}
C \sin 2 \theta_{k} \\
-C \sin 2 \theta_{k} \\
2 C \cos 2 \theta_{k}
\end{array}\right]^{-1}\left[\begin{array}{l}
\sigma_{x x} \\
\sigma_{y y} \\
\tau_{x y}
\end{array}\right]
$$

Where $u_{r}^{k}$ and $u_{\theta}^{k}$ are, respectively, the radial and tangential displacements; $k$ is the number of measured points; $A, B$ and $C$ are the calculated coefficients depending on the material elastic constants, the hole dimensions (diameter and depth) and the radial position around the blind hole; $\theta_{k}$ is the cylindrical coordinate of the measurement point and $\sigma_{x x}, \sigma_{y y}$ and $\tau_{x y}$ are stress components in Cartesians coordinates. When optical techniques are used the number of measured points can be selected from the information contained in the recorded image, this way the residual stresses can be obtained from the hole edge until the image border.

The $A, B$ and $C$ coefficients can be obtained by different ways: experimental, analytical and numerical determination. The experimental methods are most accurate ones, however are time 
consuming when compared with analytical and numerical methods, more expensive and restrict because they only can be used for a certain hole geometry and strain gage rosette. The analytical methods are based in many simplifications that, in certain cases, don't replicate the reality. So, they can only be used for the case of uniform residual stresses. The numerical methods are more generic, they can simulate any field of residual stresses, less expensive than experimental ones, it is possible to determine coefficients for different hole-drilling techniques: incremental strain, power series, integral method, average stress and ASTM E837 method. In this work a numerical determination of calibration coefficients was used.

\section{NUMERICAL DETERMINATION OF CALIBRATION COEFFICIENTS}

The main goal in this simulation it was establishing a model that it could be used for determination of calibration coefficients for different hole-drilling techniques with good accuracy. Thus, a three-dimensional finite element model was used for the numerical determination of the calibration coefficients following the procedure presented by Lu [14] and Schajer [15], using brick, homogeneous and isotropic elements in the ANSYS $®$ FEM code. A three-dimensional finite element mesh, with 5525 parametric brick elements (SOLID185) was used, as represented in fig. 2 [7].

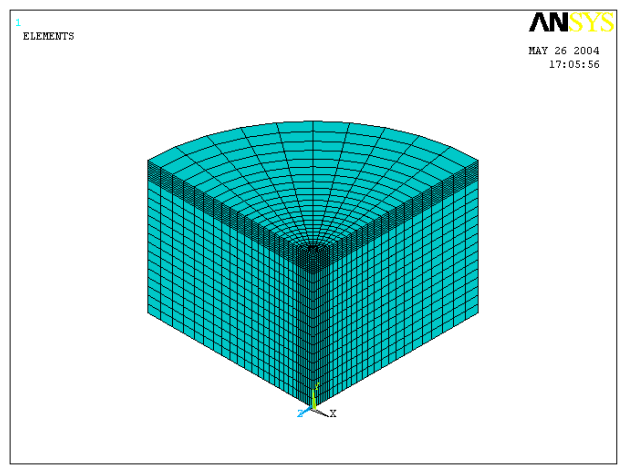

Fig. 2 FEM mesh of the three-dimensional model.

To test the described experimental methodology a calibration specimen was made in aluminum alloy (1050). This alloy (with $90 \%$ of $\mathrm{Al}$ ) was chosen because is readily available and well characterized. The material properties and geometric parameters used in this numerical simulation are shown in table 1.

Table 1 - Material, geometric and load conditions.
\begin{tabular}{ccccc}
$\sigma[\mathrm{MPa}]$ & $E[\mathrm{MPa}]$ & $v$ & $r_{0}[\mathrm{~mm}]$ & $h[\mathrm{~mm}]$ \\
\hline 100 & $7.0 \times 10^{4}$ & 0.3 & 1.0 & 0.5
\end{tabular}

where $\sigma$ is the specified residual stress, $E$ and $\nu$ are the elastic modulus and the Poisson ratio of material, $r_{0}$ and $h$ are the hole radius and depth.

Two different residual stresses states were considered.

a) Equi-biaxial: $\sigma_{x x}=\sigma_{y y}=\sigma, \tau_{x y}=0$, the correspondent stresses in cylindrical coordinates are: $\sigma_{r r}=\sigma_{\theta \theta}=\sigma, \tau_{r \theta}=0$. This drilling condition is equivalent to a uniform pressure on the surface of hole and it is represented on the left side of fig. 3 (a). 
b) Pure shear: $\sigma_{x x}=-\sigma_{y y}, \tau_{x y}=0$, the correspondent stresses in cylindrical coordinates are: $\sigma_{r r}=\sigma \cos 2 \theta, \sigma_{\theta \theta}=-\sigma \cos 2 \theta, \tau_{r \theta}=-\sigma \sin 2 \theta$. After the hole drilling this stress distribution change to an harmonic distribution of radial stresses; being $\sigma_{r r}=-\sigma \cos 2 \theta$ and a shear stress $\tau_{r \theta}=\sigma \sin 2 \theta$, both acting on the hole edge, as can be seen on the right side of fig. 3 (b). The signals of the stresses change due to stress release after the hole drilling.

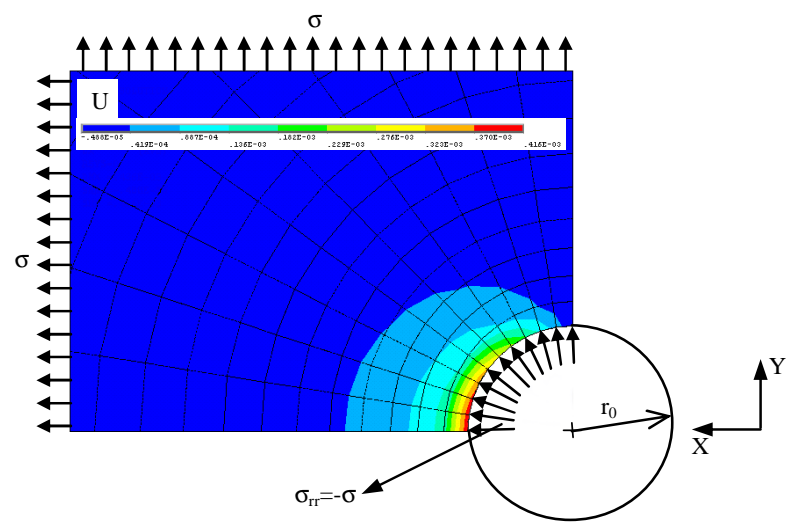

(a)

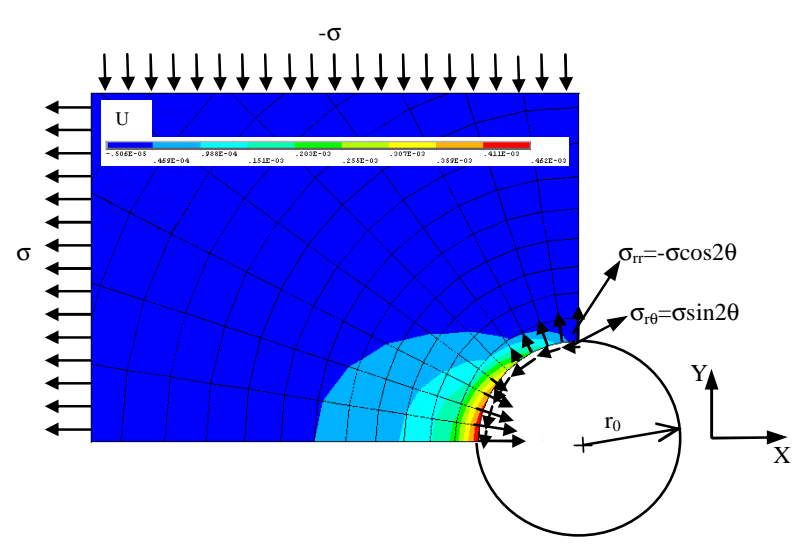

(b)

Fig.3 Three-dimensional FEM model for the calibration coefficients determination: (a) equi-biaxial stresses -

$$
\sigma_{x x}=\sigma_{y y}=\sigma, \tau_{x y}=0 ;(b) \text { pure shear stresses }-, \sigma_{x x}=-\sigma_{y y}, \tau_{x y}=0 .
$$

The calibration coefficients were computed with the expressions presented by $\mathrm{Wu}$ [10], which, for the case in analysis lead to:

$$
\begin{aligned}
& A\left(E, v, r_{0}, r, \frac{h}{d_{0}}\right)=\frac{u_{r}(r, \theta)}{2 \sigma} \\
& B\left(E, v, r_{0}, r, \frac{h}{d_{0}}\right)=\frac{u_{r}(r, \theta)}{2 \sigma \cos 2 \theta} \\
& C\left(E, v, r_{0}, r, \frac{h}{d_{0}}\right)=\frac{u_{\theta}(r, \theta)}{2 \sigma \sin 2 \theta}
\end{aligned}
$$

The calibration coefficients are dimensionless and were computed for a hole with $2 \mathrm{~mm}$ in diameter and $0.5 \mathrm{~mm}$ depth, at a radial distance of $1.2 r_{0}$ from hole center. Table 2 summarizes the values of calibration coefficients that were obtained.

\begin{tabular}{ccc}
\multicolumn{3}{c}{ Table 2 } \\
\hline A & Calibration coefficients. \\
\hline $4.64 \times 10^{-6}$ & $5.88 \times 10^{-6}$ & $3.82 \times 10^{-6}$ \\
\hline
\end{tabular}

The process was repeated for different diameters: 1,8, 2, and 2,2 $\mathrm{mm}$. 


\section{RING AND PLUG SPECIMEN}

To obtain well known residual stress fields an aluminum shrink-fit ring and plug was chosen. This specimen has a closed form solution for the residual stresses, and relatively simple stress distribution. In the plug the stress is constant, in the ring only depends on the radial position. On the other hand, this type of specimen provides the full range of biaxial stress states: in the ring near the interface, $\sigma_{\theta}$ is positive and $\sigma_{\mathrm{r}}$ is negative, near the outer edge the stress state is nearly uniaxial, (since $\sigma_{\mathrm{r}}$ goes to zero), in the plug, the stress state is equi-biaxial, $\sigma_{\theta}=\sigma_{\mathrm{r}}$. On the other hand, the residual stresses in specimen are uniform in depth, thus it is possible to use the ASTM standard [1]. Therefore, with only one specimen it is possible to demonstrate the ability of the optical technique on three different residual stress conditions [16], and several measurements could be performed.

The nominal dimensions of the specimen used were: for the exterior and interior diameter of the ring, respectively, $100 \mathrm{~mm}$ and $50 \mathrm{~mm}$. The thickness of both elements was of $13 \mathrm{~mm}$ and they were assembled with a radial interference of $0.05 \mathrm{~mm}$.

To control the assemblage conditions two strain gages were bonded at the same radial distance $(30 \mathrm{~mm})$ but oriented in two orthogonal directions (tangential and radial).For assembly, the plug was cooled in liquid nitrogen $\left(-160^{\circ} \mathrm{C}\right)$ and the ring heated $\left(40{ }^{\circ} \mathrm{C}\right)$ which leaves approximately $0.2 \mathrm{~mm}$ clearance between them. Fig. 4 represents the apparatus used in the assembly control.

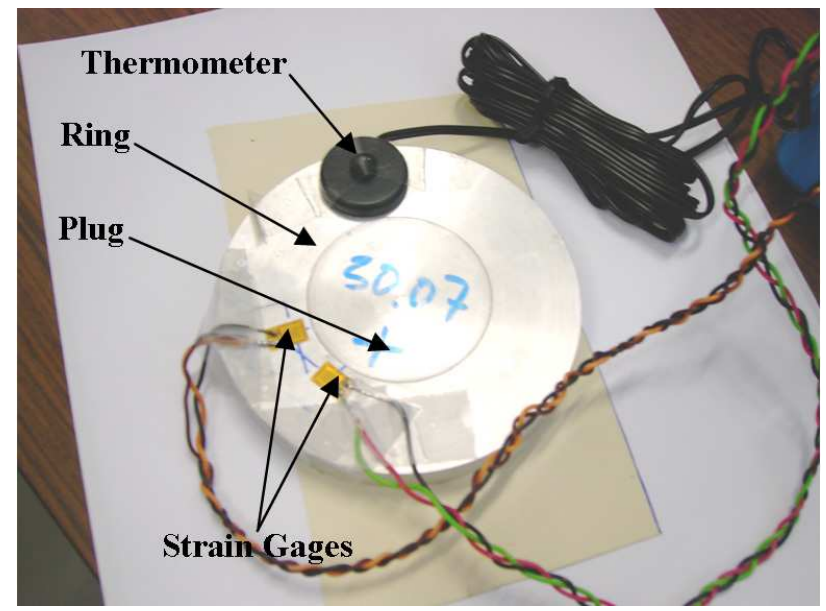

Fig.4 Apparatus used to make de assembly.

The stress distribution of the ring and plug can be easily calculated using Lamé equations [17]. First, the pressure $\mathrm{p}$ applied due to the interference is obtained by:

$p=\frac{E \delta}{2 R_{i}} \frac{\left(R_{0}^{2}-R_{i}^{2}\right)}{R_{0}^{2}}$

Were $\mathrm{p}$ is taken as positive, and $\delta$ is the radial interference between the ring and plug. In the plug the stress are equal to the pressure in the radial and angular directions. For the ring, the stresses in the same directions are calculated with:

$\sigma_{r}=\frac{p R_{i}^{2}}{R_{0}^{2}-R_{i}^{2}}\left(1-\frac{R_{0}^{2}}{r^{2}}\right)$ 


$$
\sigma_{\theta}=\frac{p R_{i}^{2}}{R_{0}^{2}-R_{i}^{2}}\left(1+\frac{R_{0}^{2}}{r^{2}}\right)
$$

In the case studied the pressure due to the radial interference $0,05 \mathrm{~mm}$ was 47.3 [MPa]. With this load the measurements performed with the strain gages allow the calculation of the average stresses $\sigma_{r}$ and $\sigma_{\theta}$, on the strain gages grid, these values are represented on the table 3.

Table 3 - Average stresses $\sigma_{r}$ and $\sigma_{\theta}$ measured by strain gages.

\begin{tabular}{cc}
\hline$\sigma_{r}[\mathrm{MPa}]$ & $\sigma_{\theta}[\mathrm{MPa}]$ \\
\hline 49.9 & -30.3 \\
\hline
\end{tabular}

These values are similar to the ones obtained with the closed form solution for the center of strain gages grids.

\section{MEASUREMENT OF RESIDUAL STRESSES}

After the specimen preparation and its stress state control, several measurements of residual stresses were made using optical techniques and the hole-drilling method. The selected points for the residual stresses measurements are represented on fig. 5.

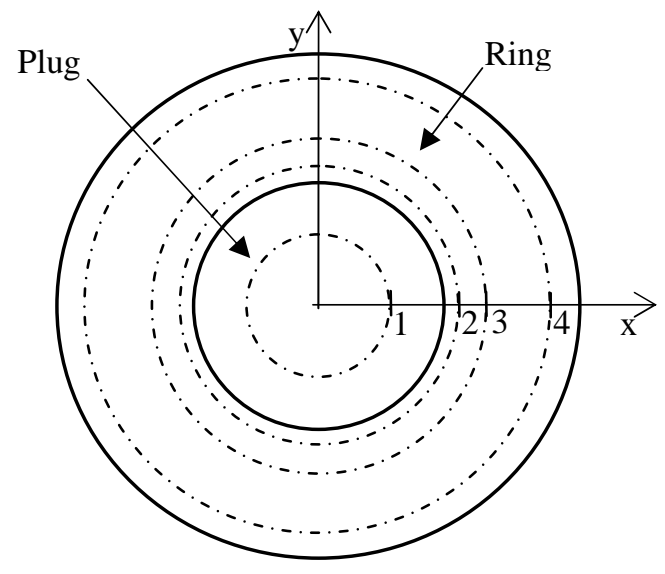

Radial distance from the center of plug:

- Point $1-15 \mathrm{~mm}$

- Point $2-29 \mathrm{~mm}$

- Point $3-32 \mathrm{~mm}$

- Point $4-45 \mathrm{~mm}$

Fig.5 Diametrical location of measurement points.

To avoid the material influence the measurements were performed in different angular positions. However, with Moiré Interferometry the grating was fixed only on a small part of the specimen and so, only two points were measured. The optical set up used to measure residual stresses with in-plane ESPI and Moiré Interferometry are schematically represented on fig. 1 (a) and (b), respectively. Fig. 6 shows details of both set-ups with the additional system for the hole drilling. 


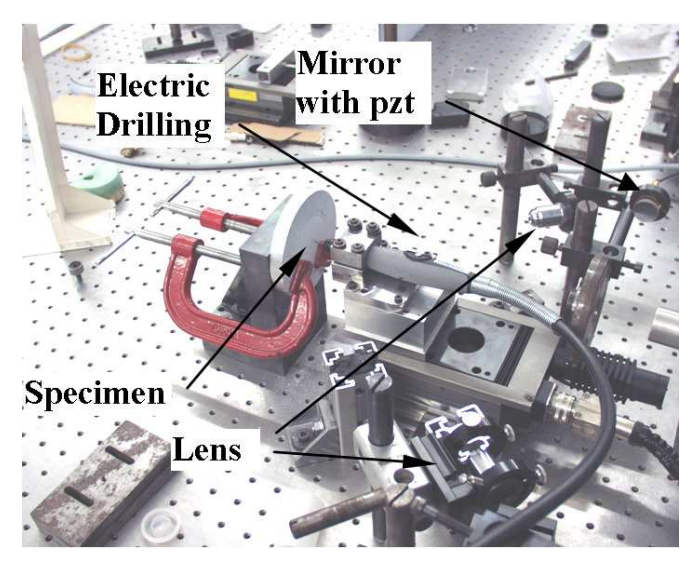

(a)

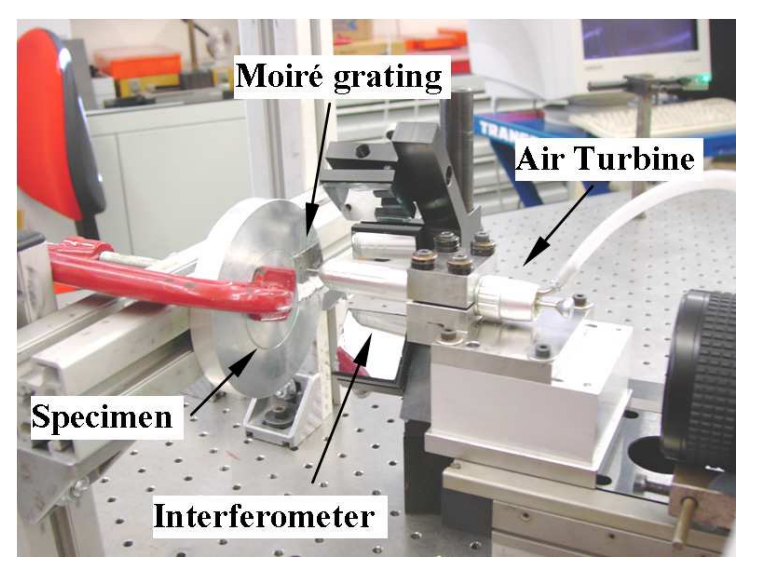

(b)

Fig.6 Apparatus to measure residual stresses using the hole-drilling technique associated to: (a) in-plane ESPI; (b) Moiré Interferometry.

To guarantee the hole concentricity and the perpendicularity between the drilling apparatus and the measured surface, a conic fixation system was fabricated with a very tight geometrical tolerance. Before the measurements were performed some testes were carried out to verify the holes geometry. After drilling the holes they were measured with an optical microscope in its main dimensions: depth and diameter.

\section{RESULTS}

To calculate the displacement field eight images should be recorded in both techniques, at different phase shift, being half of them before and the remaining after hole drilling. In ESPI the displacement field is calculated by subtracting the speckle phase maps obtained before and after strain relaxation. One of these measurements, obtained after the hole had been drilled, is presented in fig. 7 (a) and Fig. 7 (b). The discontinuities in fig. 7 (a) are due to the phase calculation algorithm and can be removed by phase unwrapping. Different solutions are available for this proposes according to the data spatial noise. The results presentation could be improved using a pseudo color presentation where the displacement intensity is codified according to a color table.

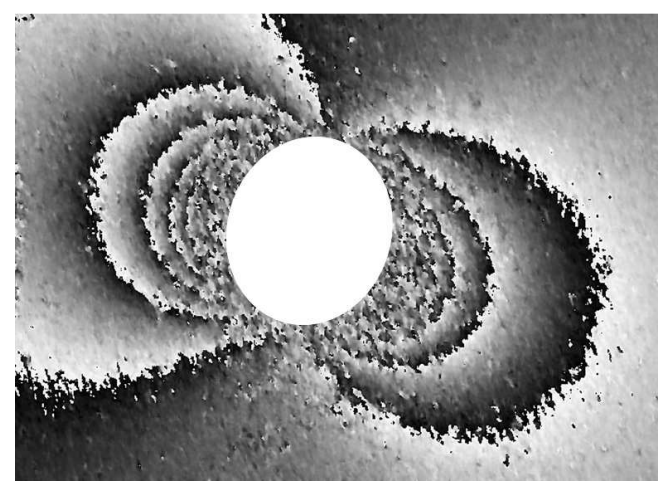

(a)

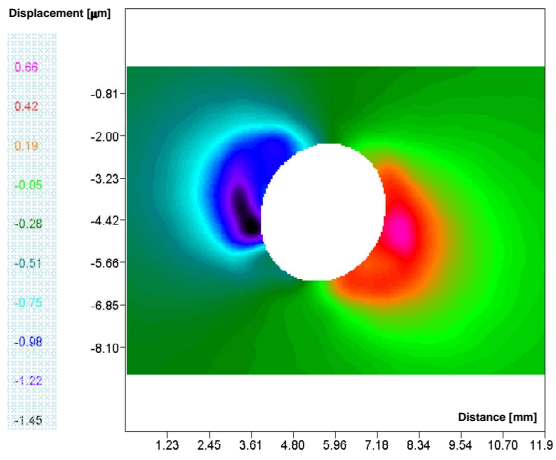

(b)

Fig.7 (a) Phase map and (b) Unwrapped phase map of displacement field. 
Using the unwrapped phase map corresponding to the displacement field and the calibration coefficients, the residual stresses can be compute using data obtained for three different points at the same radial distance from the hole center. The results presented in this work were obtained using three points placed according to fig. 8 .

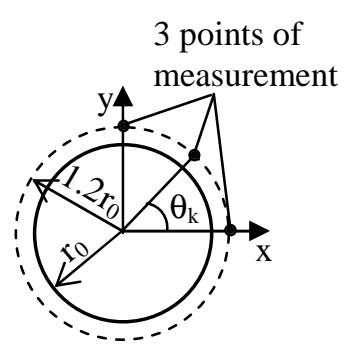

Fig.8 Points of measurement.

Where $r_{0}$ is the hole radius and $\theta_{\mathrm{k}}$ is the angular position of the measurement point. The residual stresses were calculated with equations (1) using the calibration coefficients computed with equations (2).

Fig. 9 summarizes the results obtained for residual stresses measurements. In this graphic presentation the solid lines represent the stresses calculated by the close form solutions given by Lamé. The experimental data is represented by dots. In all the cases the error was less than $15 \%$, most of the measurements were around $5 \%$ of error.

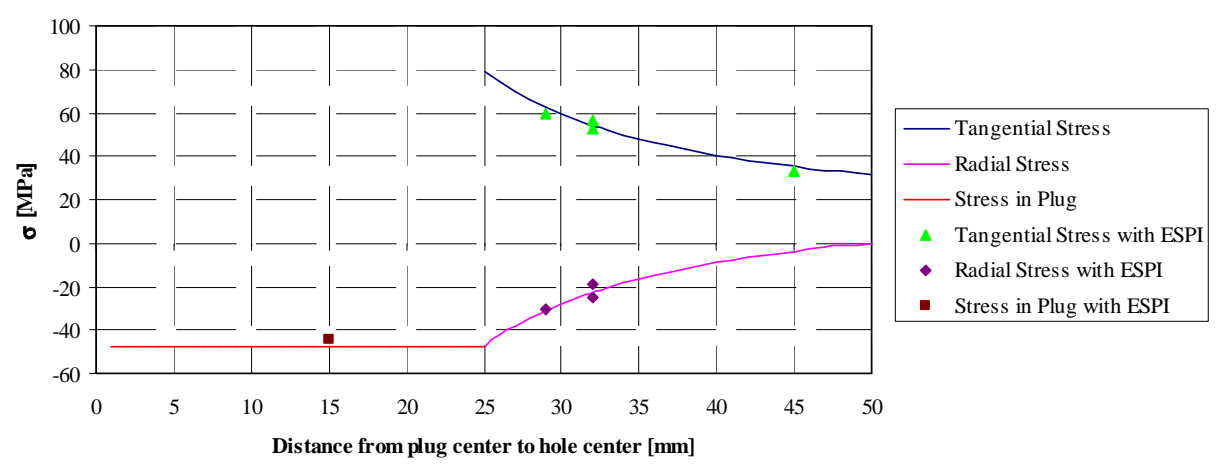

Fig.9 Closed form solution and experimental measurements with ESPI.

For the Moire Interferometry set up only the phase modulation device differs from the previous one. In this case the $90^{\circ}$ phase shift between each image was performed by rotation of a glass plate with parallel faces, instead of using a linear displaceable mirror. It should be mentioned that noisy recordings may be obtained if the grid is not properly bonded or has poor quality. Low contrast or poor reflectivity are the most important problems with the grid replication, meanwhile a constant glue thickness should be achieved. To obtain the phase maps, filtering and smoothing algorithms should be involved to deal with noisy data. In fig. 10 (a) and (b) an example of the phase map around the hole and the computed displacement field after image processing (unwrapping) are presented. 


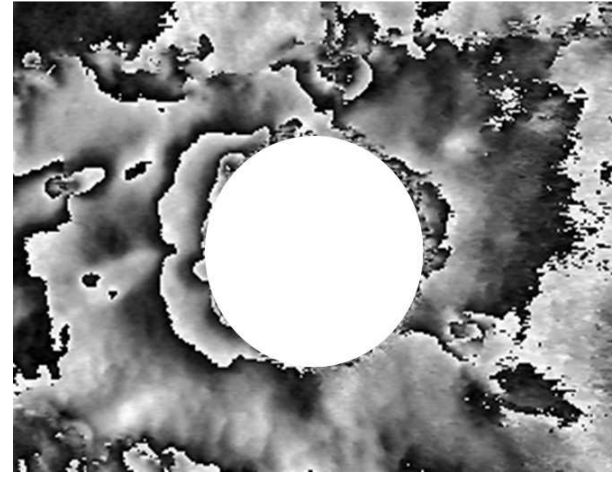

(a)

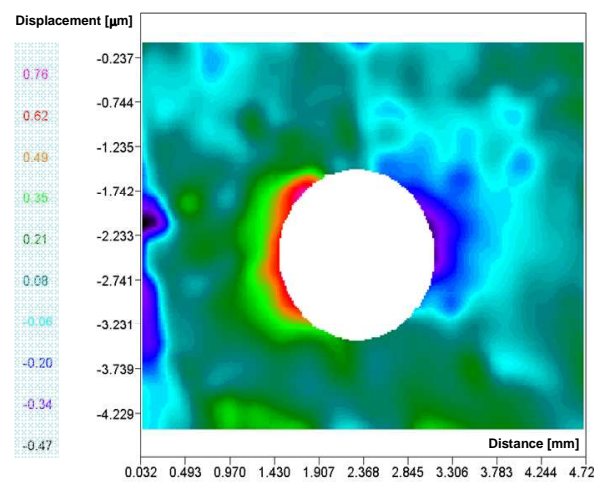

(b)

Fig. 10 (a) Phase map and (b) Unwrapped phase map of displacement field.

The procedure to compute the residual stresses using the unwrapped phase map of displacement field was the same described for ESPI technique. The results obtained after two measurements in the specimen with Moiré interferometry are presented in the fig. 11 . In this case only two measurements were made due to the size of the grid used and the necessary distance between holes to avoid influence between them. In all the measurements an error less then $17 \%$ was obtained.

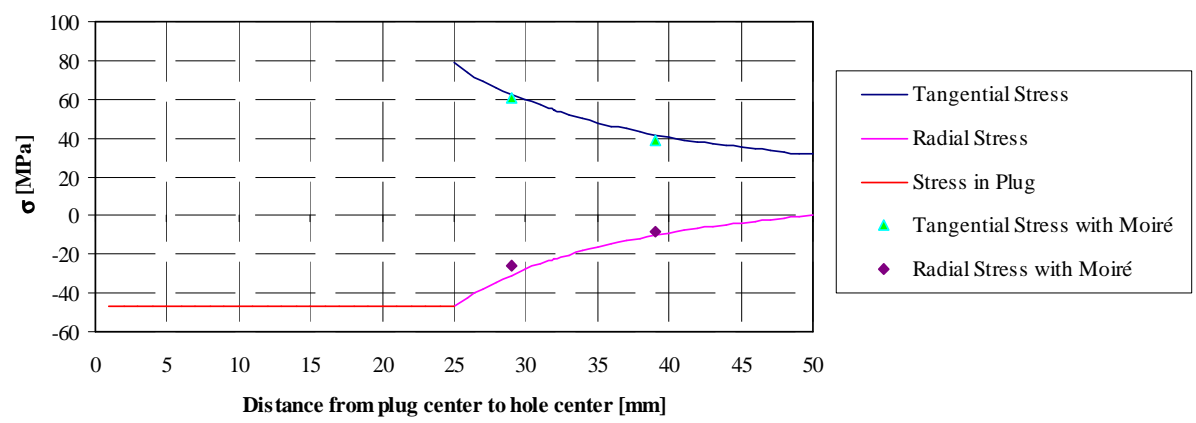

Fig. 11 Closed form solution and experimental measurements with Moiré.

All the holes were drilled in four incremental steps according to ASTM standard. They were circulars with a diameter between $2 \mathrm{~mm}$ and $2.1 \mathrm{~mm}$. Some holes were chosen to cut and verified in its sectional geometry, the hole bottom was flat and there was a perpendicularity between the bottom and wall directions of the hole.

\section{CONCLUSIONS}

In this work an experimental methodology for residual stress assessment is presented using optical techniques with the hole drilling method. These methods were tested with a well known residual stress field in a ring and plug specimen. The experimental results obtained with in-plane ESPI and Moiré Interferometry are in good agreement with the closed form solution. These optical techniques are a very interesting alternative to the traditional holedrilling method with strain gages, and present some advantages, it is a global measurement, has better resolution and allows measurements closer to the hole edge. Image processing algorithms are available to convert the displacement data obtained to values of residual stresses through a series of calibration coefficients obtained by FEM simulation. 


\section{REFERENCES}

[1] ASTM, ASTM E837-01 e1 Standard test method for determining residual stresses by the hole-drilling strain-gage method, Section 3, 03.01, 2001.

[2] Nelson, D. V., Makino, A. Schmidt, T. Residual stress determination using hole drilling and 3D image correlation, Experimental Mechanics, Vol. 46, n. 1, pg. 31-38, 2006.

[3] Min, Y., Hong, M., Xi, Z., Lu, J. Determination of residual stress by use of phase shifting moiré interferometry and hole-drilling method, Optics and Lasers in Engineering, 44, pg. 68-79, 2006.

[4] Schajer, G. S. and Steinzig, M. Full-field calculation of hole drilling residual stresses from electronic speckle pattern interferometry data, Experimental Mechanics, Vol. 45, n. 6, pg. 526-532, 2005.

[5] Post, D.; Han, B.; Ifju, P. High Sensitivity Moiré: Experimental Analysis for Mechanics and Materials. Springer Verlag, 1997.

[6] Jones, R., Leendertz, J. A. Elastic constant and strain measurement using a three beam speckle pattern interferometer. Journal of Physics E: Scientific Instruments, Vol. 7, pg. 653-657, 1974.

[7] Ribeiro, J., Vaz, M., Piloto, P. e Monteiro, J. Técnicas de Medição de Tensões Residuais. VI Encontro Nacional da Associação Portuguesa de Análise Experimental de Tensões (APAET), nos Proc., Ponta Delgada, 2005.

[8] Nelson, D. V. and Makino, A. The Holographic-hole Drilling Method for Residual Stress Determination, Optics and Lasers in Engineering, 27, pg. 3-23, 1997.

[9] Nelson, D. V. and McCrickerd, J. T. Residual-stress determination through combined use of holographic interferometry and blind hole drilling. Experimental Mechanics, 26, pg. 371-378, 1986.

[10] Wu, Z.; Lu, J.; Han, B. Study of Residual Stress Distribution of Moiré Interferometry and Incremental Hole Drilling. Journal of Applied Mechanics, Vol. 65, pg. 837-850, 1998.

[11] Creath, K. and Schmit, J. N-point Spatial Phase-measurement Techniques for Nondestructive Testing, Optics and Lasers in Engineering, 24, pg. 365-379, 1996.

[12] $\mathrm{Wu}, \mathrm{Z}$., Détermination des contraintes résiduelles par interférométrie de Moiré et méthode de perçage du trou incrémental, Thèse présentée pour l'obtention du grade de Docteur, Université de Technologie de Troyes, Troyes, 1998.

[13] Post, D., Han, B., Ifju, P., High Sensitivity Moiré - Experimental Analysis for Mechanics and Materials, Edited Springer-Verlag, New York, 1994.

[14] Lu, J.; James, M. R. and others. Handbook of Measurement of Residual Stresses. Society for Experimental, Inc., Edited by Jian Lu, 1996.

[15] Schajer, G. S. Application of Finite Element Calculations to Residual Stress Measurements. J. Eng. Mater. Tech., 103, 4, 1981, pg. 157-163.

[16] Steinzig, M., Hayman, G., Prime, M. Verification of a Technique for Holographic Residual Stress Measurement. ASME Pressure Vessels and Piping Conference, 
Published in Residual Stress Measurement and General Non-destructive Evaluation, Vol. 429, Atlanta, 2001.

[17] Lamé, M. G., 1852, Leçons sur la Théorie Mathématique de l'Élasticité des Corps Solids, bachelier, Paris. 